\title{
Triple Cohomology of Algebras and Two Term Extensions*
}

\author{
Dedicated to Professor Atuo Komalu for his 60th birthday
}

By

\begin{abstract}
Nobuo Shimada, Hiroshi Uehara**, Frank Brenneman ${ }^{\dagger}$ and Akira IwAI ${ }^{\dagger \dagger}$
\end{abstract}

\section{Introduction}

It has been recognized that the notion of triple [12] (or monad [8]) provides a unified simplicial method for defining homology and cohomology in categorical setting (Godement [15], Huber [19], Eilenberg-Moore [12], Dold-MacLane-Oberst [8], Beck [5]).

It has been shown that many known, classical or special, (co)homology theories of groups, modules and algebras (EilcnbergMacLane [10], Cartan-Eilenberg [6], Hochschild [17], Harrison [16], Shukla [27], etc.) are triple cohomologies (Barr-Beck [1], Barr [2], [3], Iwai [20]).

In the former announcement [26], we treated triple cohomologies viewing them as derived functors (in a functor category) in the sense of relative homological algebra [11], [25]. Since then such interpretations have appeared (Dold-MacLane-Oberst [8], Dubuc [9]). Therefore we will not discuss this subject here.

We will treat the calculation or interpretation of triple cohomology of an algebra with coefficients in a module [5]. The 0th

Received July 7, 1969.

* This work is a revised version of the previously announced report [26] and was partially supported by the Research Foundation of the Oklahoma State Unive'rsity while N. Shimada was a visiting professor (1966 67).

** Department of Mathematics and Statistics, Oklahoma State University. This work is partially supported by the National Science Foundation research grant GP-9585.

$\dagger$ Department of Mathematics, Lehigh University. F. Brenneman was supported by the National Aeronautics and Space Administration as a NASA Trainee ai the Oklahoma State University.

†† Yoshida College, Kyoto University. 
and 1st cohomology groups $H^{0}$ and $H^{1}$ (the dimension indices in triple cohomology being one less than usual) were discussed by J. M. Beck in his dissertation [5]. The purpose of the present paper is to interpret the second cohomology, $H^{2}(A, M)$, of an algebra $A$ with coefficients in an $A$-module $M$ as the set of equivalence classes after Yoneda [29] of two term extensions of $A$ by $M$ (see $\S 3$, Lichtenbaum-Schlessinger [23] or Gerstenhaber [13] for two term extensions).

Our interpretation appears to be more direct than those through classical obstruction theory for algebra extensions (MacLane [24], [10], Hochschild [18], Shukla [27], Barr [4]) and suggests a close relationship between $H^{n}$ and $n$ term extensions for $n>2$ (see $§ 4$ ). In fact, such an interpretation of $H^{n}$ has been obtained by A. Iwai, one of the present authors, and is to appear in his subsequent paper [21].

In the sequel we choose and deal with a specific category, the category of Lie algebras over a commutative ground ring. The argument is functorial, at least in substance, so is applicable to other categories of algebraic systems with tripleable underlying object functors [5].

The authors are indebted to Dr. Satoshi Suzuki for his critical reading of the manuscript.

\section{$\S 1 . \quad$ Preliminaries}

Recall the notion of cotriple [12]. A cotriple $\boldsymbol{G}=(G, \varepsilon, \delta)$ in a category $\mathcal{Q}$ consists of a (covariant) functor $G: \mathcal{Q} \rightarrow \mathscr{Q}$ and natural transformations $\varepsilon: G \rightarrow I_{Q} \quad\left(I_{Q}\right.$ denotes the identity functor) and $\delta: G \rightarrow G^{2}=G \circ G$ satisfying

$$
\begin{aligned}
& \varepsilon G \circ \delta=G \varepsilon \circ \delta=1_{G} \quad \begin{array}{l}
\left(1_{G}\right. \text { denotes the identity natural } \\
\text { transformation }),
\end{array} \\
& G \delta \circ \delta=\delta G \circ \delta,
\end{aligned}
$$

where $\varepsilon G$ means the natural transformation defined by

$$
\varepsilon G(A)=\varepsilon(G(A)): G^{2}(A) \rightarrow G(A), \quad \text { for } \quad A \in \mathrm{obC},
$$

and similarly for $G \varepsilon, G \delta$, and $\delta G$. Triples are defined dually.

A cotriple comes usually from an adjoint pair of functors $(S, U)$, 
$U: \mathbb{Q} \rightarrow \mathcal{C}$ and $S: \mathcal{C} \rightarrow \mathfrak{Q}$ in the following way. Let $S$ be the left adjoint of $U$ (notation $S \dashv U$ ). This means that there exists a natural equivalence $\lambda$ between the Hom set functors:

$$
\lambda(C, A): \operatorname{Hom}_{\mathfrak{Q}}(S(C), A) \underset{\Im}{\approx} \operatorname{Hom}_{\mathcal{C}}(C, U(A))
$$

for $C \in \mathrm{ob} \mathcal{C}, A \in \mathrm{ob} C$. Define natural transformations $\varepsilon$ and $\eta$ by

$$
\begin{array}{ll}
\varepsilon(A)=\lambda^{-1}\left(1_{U(A)}\right): S U(A) \rightarrow A, & \text { for } \quad A \in \mathrm{ob} \mathcal{Q}, \\
\eta(C)=\lambda\left(1_{S(\mathcal{C})}\right): C \rightarrow U S(C), & \text { for } \quad C \in \mathrm{obC},
\end{array}
$$

with abbreviation $\lambda=\lambda(U(A), A)$ resp. $\lambda=\lambda(C, S(C))$. Then we have

$$
\lambda(\rho)=U(\rho) \circ \eta(C), \quad \text { for } \quad \rho \in \operatorname{Hom}_{\mathbb{Q}}(S(C), A),
$$

with $\lambda=\lambda(C, A)$ and

$$
\begin{aligned}
& \varepsilon S \circ S \eta=1_{S}: S \rightarrow S U S \rightarrow S, \\
& U \varepsilon \circ \eta U=1_{U}: U \rightarrow U S U \rightarrow U .
\end{aligned}
$$

It follows that the adjoint pair $(S, U)$ yields a cotriple $(S U, \varepsilon, S \eta U)$ in $\mathcal{Q}$ (dually a triple $(U S, \eta, U \varepsilon S)$ in $\mathcal{C}$ ).

Conversely, given a cotriple $(G, \varepsilon, \delta)$ in $\mathcal{Q}$. It is known ([12], [22]) that there exist a category $\mathcal{C}$ and an adjoint pair of functors $(S, U), U: \mathbb{Q} \rightarrow \mathbb{C}, S: \mathcal{C} \rightarrow \mathbb{Q}$ with $S \dashv U$, irducing the cotriple $(G, \varepsilon, \delta)$ as above.

From now on we assume that all categories considered are pointed (i.e. have zero objects) with kernels and all functors are also pointed (i.e. $T(0)=0$ ), unless otherwise stated.

Given an adjoint pair of functors $U: \mathbb{Q} \rightarrow \mathcal{C}$ and $S: \mathcal{C} \rightarrow \mathbb{Q}$ with $S \dashv U$ which induces a cotriple $\boldsymbol{G}=(G, \varepsilon, \delta)$ in $\mathcal{Q}$. There is naturally defined a simplicial object $G_{*}=\left\{G_{n} ; n \geq 0\right\}$ in the category of endofunctors : $\mathfrak{Q} \rightarrow \mathbb{Q}$, with $G_{n}=G^{n+1}=G \circ \cdots \circ G((n+1)$-fold iterated composition of $G$ ) and

$$
\begin{array}{ll}
\varepsilon_{n}^{i}=G^{i} \varepsilon G^{n-i}: G_{n} \rightarrow G_{n-1}, & 0 \leq i \leq n \text { (face operator), } \\
\delta_{n}^{i}=G^{i} \delta G^{n-1}: G_{n} \rightarrow G_{n+1}, & 0 \leq i \leq n \text { (degeneracy operator). }
\end{array}
$$

Here we have the usual commutation rule:

$$
\varepsilon^{i} \delta^{j}= \begin{cases}\delta^{j-1} \varepsilon^{i} & (i<j) \\ \text { identity } & (i=j \text { or } j+1) \\ \delta^{j} \varepsilon^{i-1} & (i>j+1)\end{cases}
$$




$$
\begin{aligned}
& \varepsilon^{i} \varepsilon^{j}=\varepsilon^{j-1} \varepsilon^{i} \quad(i<j) \\
& \delta^{i} \delta^{j}=\delta^{j-1} \delta^{i} \quad(i \leq j)
\end{aligned}
$$

with abbreviations $\varepsilon^{i}=\varepsilon_{n}^{i}$ and $\delta^{j}=\delta_{n}^{j}$. For any object $A$ in $\mathscr{Q}$ the corresponding simplicial object $G_{*}(A)=\left\{G_{n}(A) ; n \geq 0\right\}$ in $\mathscr{Q}$ is called the standard simplicial complex over $A$ associated with the cotriple $G$. And the augmented simplicial complex $G_{*}^{+}(A)=\left\{G_{n}(A) ; n \geq-1\right\}$ with natural augmentation $\varepsilon(A): G_{0}(A) \rightarrow G_{\sim 1}(A)=A$, i.e. the sequence

$$
\cdots \cdots G_{n}(A) \underset{\overrightarrow{\varepsilon^{n}}}{\stackrel{\varepsilon^{0}}{\stackrel{\vec{b}}{\rightarrow}}} G_{n-1}(A) \cdots \cdots \stackrel{\rightarrow}{\rightarrow} G_{1}(A) \underset{\varepsilon^{1}}{\stackrel{\varepsilon^{\prime \prime}}{\rightarrow}} G_{0}(A) \stackrel{\varepsilon}{\rightarrow} A,
$$

together with degeneracy operators, is called the standard simplicial resolution of $A$.

The following lemma is a slight modification of Theorem in [20].

Lemma 1.4. Suppose that $U(A)$ is an abelian group object in $\mathrm{C}$ for every object $A$ in $\mathcal{A}$. Then the augmented chain complex $U G_{*}^{+}(A)$ with differential $d_{n}(A)=\sum_{i=0}^{n}(-1)^{i} U \varepsilon^{i}(A)(n>0)$ and augmentation $U \varepsilon(A)$ is acyclic. Precisely, there exists a contracting homotopy $s_{n}(A)$ : $U G_{n}(A) \rightarrow U G_{n+1}(A)(n \geq-1)$ such that $U \varepsilon \circ S_{-1}=1$ and

$$
d_{n \dashv 1} s_{n}+s_{n-1} d_{n}=1 \quad(n \geq 0) .
$$

Proof. Define morphisms $t_{n}(A): U G_{m}(A) \rightarrow U G_{m}(A)$ and $u_{n}(A)$ : $U G_{m}(A) \rightarrow U G_{m \mid 1}(A)$, for $0 \leq n \leq m$, as follows :

$$
\begin{aligned}
& t_{0}=1, \quad u_{-1}=0, \quad u_{0}=0 \\
& t_{n}=\left(1-U \delta^{0} \varepsilon^{1}\right)\left(1-U \delta^{1} \varepsilon^{2}\right) \cdots\left(1-U \delta^{n-1} \varepsilon^{n}\right) \quad(n \geq 1) \\
& u_{n}=t_{0} \circ U \delta^{0}-t_{1} \circ U \delta^{1}+\cdots+(-1)^{n-1} t_{n-1} \circ U \delta^{n-1} \quad(n \geq 1) \text {. }
\end{aligned}
$$

Then we have

$$
\begin{aligned}
& U \varepsilon^{i} \circ t_{n}=\left\{\begin{array}{lr}
t_{n-1} \circ d_{n} & (i=0) \\
0 & (0<i \leq n)
\end{array}\right. \\
& 1-t_{n}=d_{n+1} u_{n}+u_{n-1} d_{n} \quad(n \geq 0) .
\end{aligned}
$$

Now define morphisms $\eta_{n}(A)=\eta U G_{n}(A): U G_{n}(A) \rightarrow U G_{n+1}(A)$, which satisfy 


$$
\begin{aligned}
U \varepsilon^{i} \circ \eta_{n} & =\left\{\begin{array}{cl}
1 . & (i=0) \\
\eta_{n-1} \circ U \varepsilon^{i-1} & (0<i \leq n+1)
\end{array}\right. \\
U \varepsilon \circ \eta_{-1} & =1 .
\end{aligned}
$$

Using these we get the required contracting homotopy :

$$
\begin{array}{ll}
s_{n}=\eta_{n} t_{n}+u_{n} & (n \geq 0) \\
s_{-1}=\eta_{-1}=\eta U . &
\end{array}
$$

Note that all these $t_{n}, u_{n}, \eta_{n}$ and $s_{n}$ are natural transformations of functors $\mathfrak{Q} \rightarrow \mathcal{C}$.

For later use, we give the following variant of the above lemma which will be similarly proved.

Lemma 1.9. Suppose that there is given another category $\mathcal{C}^{\prime}$ and a functor $U^{\prime}: \mathcal{C} \rightarrow \mathbb{C}^{\prime}$ such that $U^{\prime} U(A)$ (but not $U(A)$ ) is an abelian group object in $\mathcal{C}^{\prime}$ for every object $A$ in $\mathfrak{Q}$. Then the augmented chain complex $U^{\prime} U G_{*}^{+}(A)$ with differential $d_{n}(A)=\sum_{i=0}^{n}(-1)^{i} U^{\prime} U \varepsilon^{i}(A)$ $(n>0)$ and augmentation $U^{\prime} U \varepsilon(A)$ is acyclic.

For the purpose of defining cotriple cohomology, we first fix an object $A$ in $\mathfrak{Q}$ and consider the comma category $(\mathfrak{Q}, A)$ [5]. By definition an object in $(\mathfrak{Q}, A)$ is a pair $(B, \gamma)$ of an object $B$ in $\mathcal{Q}$ and a morphism $\gamma: B \rightarrow A$ in $\mathcal{Q}$, and a morphism $(B, \gamma) \rightarrow\left(B^{\prime}, \gamma^{\prime}\right)$ is such a commutative diagram:

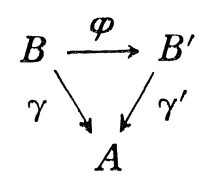

An object $(B, \gamma)$ in $(\mathfrak{Q}, A)$ will be offen denoted by $B \rightarrow A$ or simply by $B$. Note that the comma category $(\mathfrak{Q}, A)$ is not pointed for $A \neq 0$, but has kernels and the terminal object $A=\left(A, 1_{A}\right)$.

An adjoint pair $\stackrel{S}{\rightarrow} \mathfrak{Q} \stackrel{U}{\rightarrow} \mathcal{C}$ induces canonically an adjoint pair $(\mathcal{C}, U(A)) \stackrel{S}{\rightarrow}(\mathcal{Q}, A) \stackrel{U}{\rightarrow}(\mathcal{C}, U(A))$ which will be denoted by the same symbols $S$ and $U$ as before, and similarly for the induced cotriple $\boldsymbol{G}=(G, \varepsilon, \delta), G=S U, \delta=S_{\eta} U$. Note that $G^{n}(A)$ is regarded canonically as an object in $(\mathfrak{Q}, A)$ with the unique morphism $G^{n}(A) \rightarrow A$ expressed by a composition of face operators. 
Now denote by $A b$ the category of abelian groups and let $T:(\mathfrak{Q}, A) \rightarrow A b$ be a contravariant functor. Then we have a cochain complex $T G_{*}(A)=\left\{T G_{n}(A) ; n \geq 0\right\}$ with differential $d^{*}(A)=\sum(-1)^{i}$ $\times T \varepsilon^{i}(A)$. Its derived groups $H^{n}\left(T G_{*}(A)\right)$ are, by definition, the cotriple cohomology groups of $A$ with initial group $H^{\circ}\left(T G_{*}(A)\right)=T(A)$. For example take an abelian group object $Y \rightarrow A$ in $(\mathcal{Q}, A)$ and define a functor $T:(\mathfrak{Q}, A) \rightarrow A b$ by $T(X)=\operatorname{Hom}_{(\mathfrak{Q}, A)}(X, Y)$. Then we have the cohomology groups $H_{G}^{n}(A, Y)=H^{\prime \prime}\left(T G_{*}(A)\right)$ with $H_{G}^{0}(A, Y)=$ $\operatorname{Hom}_{(\mathfrak{Q}, A)}(A, Y)$.

\section{§. Lie Algebras and Cotriples}

Let $K$ be a commutative ring with unit which we fix as ground ring. By a $K$-Lie algebra we mean a $K$-module $\Gamma$ with a $K$-bilinear product $[x, y] \in \Gamma$ such that

$$
\begin{aligned}
& {[x, x]=0,} \\
& {[x,[y, z]]+[y,[z, x]]+[z,[x, y]]=0}
\end{aligned}
$$

for $x, y, z \in \Gamma$. By a $\Gamma$-module for a $K$-Lie algebra $\Gamma$ we mean a $K$-module $M$ with left operation of $\Gamma$ on $M$ such that $[x, y] m=$ $x y m-y x m$ for $x, y \in \Gamma, m \in M$.

Let $\mathcal{L}$ be category of all $K$-Lie algebras with obvious morphisms, and ${ }_{\Gamma} \Upsilon \mathcal{C}$ be the category of all $\Gamma$-modules. Then every abelian group object in the comma category $(\mathcal{L}, \Gamma)$ is known to be of the form of the split extension $\Gamma * M$ of $\Gamma$ by a $\Gamma$-module $M$. This may be called also the idealization of a $\Gamma$-module $M$ and is defined as a direct product $\Gamma \times M$ of $K$-modules with bracket product

$$
[(x, m),(y, n)]=([x, y], x n-y m)
$$

for $x, y \in \Gamma$ and $m, n \in M$. With the injection $m \rightarrow(0, m)$ and the projection $(x, m) \rightarrow x$, and regarding $M$ as an abelian Lie algebra, we have a split exact sequence

$$
0 \rightarrow M \rightarrow \Gamma * M \rightarrow \Gamma \rightarrow 0
$$

in $\mathcal{L}$ (also in $(\mathcal{L}, \Gamma)$ ).

Denote by $\operatorname{Ab}(\mathcal{L}, \Gamma)$ the full subcategory of $(\mathcal{L}, \Gamma)$ formed of all abelian group objects. Then we have an equivalence of categories 


$$
\operatorname{Ab}\left(\mathcal{L}^{\prime}, \Gamma\right) \underset{\Theta}{\stackrel{\text { Ker }}{\rightleftarrows}} \Gamma
$$

where $\operatorname{Ker}$ denotes the kernel functor and $\Theta(M)=\Gamma * M$ [5].

For a $\Gamma$-module $M$ and an object $(L, \gamma)$ in $(\mathcal{L}, \Gamma)$, a $K$-derivation (or simply derivalion) $f: L \rightarrow M$ is defined as a $K$-linear map such that

$$
f[x, y]=\gamma(x) f(y)-\gamma(y) f(x) .
$$

The set of all such $K$-derivations $f: L \rightarrow M$ forms an abelian group dewoted by $\operatorname{Der}_{M}(L, \gamma)$ (or simply by $\operatorname{Der}_{M}(L)$ ), and it defines the derivation functor $\operatorname{Der}_{M}:(\mathcal{L}, \mathrm{T}) \rightarrow \mathrm{Ab}$. As is well known, there is a canonical isomorphism

$$
\operatorname{Der}_{M}(L) \approx \operatorname{Hom}_{\left(\mathcal{L}, \mathrm{I}^{\mathrm{V}}\right)}(L, \Theta(M))
$$

Now we consider the cotriple cohomology $H_{G}^{\prime \prime}(\Gamma, \Theta(M))=$ $H^{n}\left(\operatorname{Der}_{M} G_{*}(\Gamma)\right)$ with respect to a cotriple $G$ in $(\mathcal{L}, \Gamma)$ as defincd at the end of $\$ 1$. For brevity we occasionally denote $H_{G}^{n}(\Gamma, \Theta(M))$ by $H_{G}^{n}(\Gamma, M)$ (or $H^{n}(\Gamma, M)$ ) and call this the $n$-th collomology group of I' with coefficients in $M$.

To calculate explicitly $H_{G}^{u}\left(\mathrm{~J}^{\prime}, M\right)$, we sliall choose some typical cotriples $G$ in $\mathcal{L}$. Take $\mathcal{L}$ for $\mathscr{Q}$ in the preceding section. Let $\mathcal{C}$ be either one of the following pointed categories: 1) the category ${ }_{K}$ ) $K$ of $K$-modules, 2) the category $S$ of pointed sets (i.e. sets with base points and base points preserving maps) and 3) the catcgory $\mathcal{S}^{\times}$of pointed sets with multiplications. More explanation is needed for the last category. An object in $\mathcal{S}^{\times}$is a pointed set $\left(X, x_{0}\right)$ with multiplication (may be non-associative) $X \times X \stackrel{\rightarrow}{\rightarrow}$ such that $x_{0} \cdot x=$ $x \cdot x_{0}=x_{0}$ for any $x \in X$ and a morphism $f:\left(X, x_{0}\right) \rightarrow\left(Y, y_{0}\right)$ is a multiplication preserving set map.

According to each of the above cases 1),2) and 3), $\mathcal{C}$ will be also denoted by $\mathcal{C}_{i}(i=1,2,3)$. Let $U_{i}: \mathcal{L} \rightarrow \mathcal{C}_{i}$ be the underlying object functor. It is clear that the $U_{i}$ are faithful and known to be tripleable in the scuse of Beck [5] for all the above cases of $\mathcal{C}$. The left adjoint $S_{i}: C_{i}-\mathcal{L}$ of $U_{i}$ will be given as follows in respective cases.

In case $\left.C_{1}={ }_{K}\right) \pi, S_{1}$ is given by the functor $L$ described in [b], 
that is, for a $K$-module $M, S_{1}(M)=L(M)$ is the quotient $K$-Lie algebra of the free non-associative algebra $A(M)=M+M \otimes M+(M \otimes M) \otimes M+M \otimes$ $(M \otimes M)+\cdots$ by the two-sided ideal generated by elements of the form $m \otimes m$ and $m_{1} \otimes\left(m_{2} \otimes m_{3}\right)+m_{2} \otimes\left(m_{3} \otimes m_{1}\right)+m_{3} \otimes\left(m_{1} \otimes m_{2}\right)$ for $m, m_{i} \in M$.

In case $\mathcal{C}_{2}=\mathcal{S}, S_{2}$ is given by $S_{2}\left(X, x_{0}\right)=L F\left(X, x_{0}\right)$ where $F\left(X, x_{0}\right)$ $=K(X) / K\left(x_{0}\right)$ is the free $K$-module generated by the set $X$ with identification $x_{0}=0$.

In case $\mathcal{C}_{3}=\mathcal{S}^{\times}$, the functor $S_{3}$ is given as follows: Let $\left(X, x_{0}\right)$ be an object in $S^{\times}$. Construct first the free $K$-module $F\left(X, x_{0}\right)$ as above and introduce in it a unique $K$-bilinear multiplication induced from the multiplication of $X$. Then the $K$-Lie algebra $S_{3}\left(X, x_{0}\right)$ is defined as the quotient of the non-associative $K$-algebra $F\left(X, x_{0}\right)$ by the two-sided ideal generated by elements of the form $x \cdot x$ and $x \cdot(y \cdot z)+y \cdot(z \cdot x)+z \cdot(x \cdot y)$.

The corresponding cotriples $\boldsymbol{G}$ and the cohomologies $H_{G}^{n}\left(\Gamma^{\top}, M\right)$ will be denoted by $\boldsymbol{G}_{i}$ and $H_{i}^{n}(\Gamma, M)$ in respective cases $i=1,2,3$.

We remark that $H_{1}^{*}(\Gamma, M)$ is the Hochschild cohomology [17] and $H_{2}^{*}(\Gamma, M)$ is related to the cohomologies of Dixmier [7] and Shukla [28]. It is known in [5] and [1] that

$$
H_{i}^{n}(\Gamma, M) \approx \begin{cases}\operatorname{Der}_{M}(\Gamma) & (n=0) \\ \operatorname{Ex}_{\imath}^{1}(\Gamma, M) & (n=1)\end{cases}
$$

for $i=1,2,3$, where $\operatorname{Ex}_{i}^{1}(\Gamma, M)$ aenotes the set of all isomorphism classes of singular $U_{i}$-split extensions of $\Gamma$ by $M$ in $\mathcal{L}$. The bijective correspondence $H^{1} \approx \mathrm{Ex}^{1}$ becomes an isomorphism of $K$-modules if we introduce a suitable Baer sum in $\operatorname{Ex}_{i}^{1}(\Gamma, M)$.

To conclude this section, we are situated in the following com mutative diagram

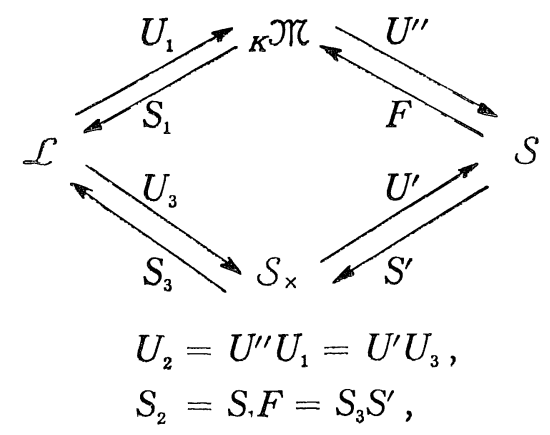


where $U^{\prime}$ and $U^{\prime \prime}$ are the forgetful functors and $S^{\prime}$ is the left adjoint of $U^{\prime}$.

\section{§3. Two Term Extensions and Main Theorem}

Let $M$ be a $\Gamma$-module which may be regarded as an abelian $K$-Lie algebra. By a $U$-split exact sequence in $\mathcal{L}$ we means a sequence in $\mathcal{L}$ of which transformation by $U$ is split exact (in $\mathcal{C}$ ).

Definition. By a two term extension of $\Gamma$ by $M$ with respect to the underlying object functor $U_{i}$ we mean a $U_{i}$-split exact sequence in $\mathcal{L}(i=1,2,3)$ :

$$
(e): \quad U \rightarrow M \stackrel{\varphi_{2}}{\rightarrow} X_{1} \stackrel{\varphi_{1}}{\rightarrow} X_{0} \stackrel{\varphi_{0}}{\rightarrow} \Gamma \rightarrow 0
$$

where $X_{0}$ operates on $X_{1}$ in the following way:

$$
\begin{aligned}
& \varphi_{2}\left(\varphi_{0}(x) m\right)=x \cdot \varphi_{2}(m), \\
& \varphi_{1}(x \cdot u)=\left[x, \varphi_{1}(u)\right], \\
& \varphi_{1}(u) \cdot v=[u, v]
\end{aligned}
$$

for $x \in X_{0}, m \in M, u, v \in X_{1}$. Moreover, in the case $i=3$ (i.e. $\mathcal{C}=S^{\times}$), we put an extra condition: there exists a set map $\beta: X_{0} \rightarrow X_{1}$ such that

$$
\begin{aligned}
& \varphi_{1} \beta=1-\sigma \varphi_{0}, \\
& \beta[x, y]=x \beta(y)-\sigma \varphi_{0} y \cdot \beta(x) \quad \text { for } \quad x, y \in X_{0},
\end{aligned}
$$

where $\sigma: \Gamma \rightarrow X_{0}$ is a morphism in $\mathcal{S}^{\times}$satisfying $\varphi_{0} \sigma=1$ (the existence of such a map $\sigma$ is ensured by the $U$-splitness of the sequence $(e)$ ).

The totality of all such two term extensions of $\Gamma$ by $M$ with respect to $U_{i}$ form a category $\mathcal{E}_{i}$ of which a morphism $(e) \rightarrow\left(e^{\prime}\right)$ is given by a commutative diagram :

$$
\begin{aligned}
& \text { (c) : } 0 \rightarrow M \stackrel{\varphi_{2}}{\rightarrow} X_{1} \stackrel{\varphi_{1}}{\rightarrow} X_{0} \stackrel{\varphi_{0}}{\rightarrow} \Gamma \rightarrow 0 \\
& \| \downarrow \psi_{1} \downarrow \psi_{0} \\
& \left(e^{\prime}\right): \quad 0 \rightarrow M \stackrel{\varphi_{2}^{\prime}}{\rightarrow} Y_{1} \stackrel{\varphi_{1}^{\prime}}{\rightarrow} Y_{0} \stackrel{\varphi_{0}^{\prime}}{\rightarrow} \Gamma \rightarrow 0
\end{aligned}
$$

where $\psi_{0}$ and $\psi_{1}$ are morphisms in $\mathcal{L}$ compatible with the operations of $X_{0}$ and $Y_{0}$ on $X_{1}$ and $Y_{1}(i=1,2,3)$. To extensions $(e)$ and $\left(e^{\prime}\right)$ 
are called equivalent (notation $(e) \sim\left(e^{\prime}\right)$ ) if they are connected by a sequence of morphisms of both directions : e.g.,

$$
(e)=\left(e_{0}\right) \leftarrow\left(e_{1}\right) \rightarrow\left(e_{2}\right) \rightarrow \cdots \leftarrow\left(e_{n}\right)=\left(e^{\prime}\right) .
$$

The set of all equivalence classes of two term extensions of $\Gamma$ by $M$ with respect to the underlying object functor $U_{i}$ is denoted by $\operatorname{Ex}_{i}^{2}(\Gamma, M)(i=1,2,3)$.

Now we can state our main theorem.

Theorem 3.5. There is a bijective correspondence

$$
H_{i}^{2}\left(I^{\prime}, M\right) \approx \operatorname{Ex}_{i}^{2}(\Gamma, M)
$$

for each $i=1,2,3$.

The proof of this theorem will be given in $\$ 5$.

We remark that, for an object $L$ in $\mathcal{L}$, the underlying $K$-module $U_{1}(L)$ and the underlying set $U_{2}(L)$ are clearly abelian group objects in ${ }_{K} \Re$ and $S$ respectively, but $U_{3}(L)$ is not so in $\mathcal{S}^{\times}$, which is the reason why we need the exceptional condition (3.4) in the definition of two term extensions with respect to $U_{3}$.

\section{\$4. Standard Two Term Extensions}

Take any one of the cotriples $\boldsymbol{G}_{i}$ in $(\mathcal{L}, \Gamma), i=1,2,3$, as before. The standard simplicial complex $G_{*}(\Gamma)$ over $\Gamma$ induces the underlying chain complex of $K$-modules, denoted by the same notation $G_{*}(\Gamma)$, with differential $d_{n}=\sum_{i=0}^{n}(-1)^{i} \varepsilon^{i}(\Gamma)(n \geq 1)$ and the augmented complex $G_{*}^{+}(\Gamma)$ is acyclic by Lemma 1.4 in cases $\mathcal{C}={ }_{K} \Re$ or $\mathcal{S}$, by Lemma 1.9 in case $c=S^{\times}$.

Let $M$ be a $\Gamma$-module. $G^{n}(\Gamma)$ operates naturally on $M$ via the canonical morphism $G^{n}(\Gamma) \rightarrow \Gamma$ (see $\$ 1$ ), so that $M$ is considered as a $G^{\prime \prime}(\Gamma)$-module for every $n \geq 0$.

A derivation 2-cocycle $f \in \operatorname{Der}_{M}\left(G^{3}(\Gamma)\right)$ is a $K$-linear map $f: G^{3}(\Gamma)$ $\rightarrow M$ and $f[x, y]=x f(y)-y f(x)$ for $x, y \in G^{3}(\Gamma)$ and $d^{*} f=f d_{3}=$ $0: G^{4}(\Gamma) \rightarrow M$. Two such cocycles $f$ and $f^{\prime}$ are $D$-cohomologous (notation $\underset{D}{\sim \sim} f^{\prime}$ ) if there exists a derivation $\omega: G^{2}(\Gamma) \rightarrow M$ and $\omega d_{2}$ $=f-f^{\prime}$.

Given a derivation 2-cocycle $f \in \operatorname{Der}_{M}\left(G^{3}(\Gamma)\right)$. We shall construct 
a $K$-Lie algebra $E_{f}$ as follows. Put $\widetilde{G}_{1}(\Gamma)=\operatorname{Ker}\left(\varepsilon^{1}: G^{2}(\Gamma) \rightarrow G(\Gamma)\right)$. Let $\widetilde{G}_{1}(\Gamma) \times M$ be the direct product of $K$-Lie algebras ( $M$ being an abelian Lie algebra). Let $I$ be the ideal of $\widetilde{G}_{1}(\Gamma) \times M$ generated by elements of the form $\left(-t_{1} d_{2} y, f\left(t_{2} y\right)\right)$ for $y \in G^{3}(\Gamma)$ (see (1.5)). Define $E_{f}$ to be the quotient $K$-Lie algebra $\widetilde{G}_{1}(\Gamma) \times M / I$. Then $E_{f}$ has a set-presentation $N(\Gamma) \times M$, where $N(\Gamma)=\operatorname{Ker}(\varepsilon: G(\Gamma) \rightarrow \Gamma)$. To see this, we define set maps

$$
\pi: \widetilde{G}_{1}(\Gamma) \times M \rightarrow N(\Gamma) \times M
$$

and

$$
\kappa: N(\Gamma) \times M \rightarrow \widetilde{G}_{1}(\Gamma) \times M
$$

by

$$
\pi(x, m)=\left(d_{1} x, m+f(\bar{x})\right)
$$

and

$$
\kappa(n, m)=(\bar{n}, m)
$$

respectively, where $\bar{x}=\eta_{n}(x)$ for $x \in U G_{n}(\Gamma)$ (see (1.7)).

Direct calculations show that

$$
\begin{aligned}
& \pi^{\prime}(I)=(0,0), \\
& \pi^{\circ}=\text { identity },
\end{aligned}
$$

and

$$
\kappa \circ \equiv \text { identity } \bmod I .
$$

It follows that $\pi$ induces a canonical one to one correspondence $E_{f} \approx N(\Gamma) \times M$ as set.

Denote by $(n, m)$ an element of $E_{f}$ for $n \in N(\Gamma)$ and $m \in M$ in this presentation. Then the $K$-Lie algebra structure of $E_{f}$ is given explicitly by

$$
\begin{aligned}
& \left(n_{1}, m_{1}\right)+\left(n_{2}, m_{2}\right)=\left(n_{1}+n_{2}, m_{1}+m_{2}+f\left(\overline{\left.\left.\overline{n_{1}}+\overline{n_{2}}\right)\right),}\right.\right. \\
& k(n, m)=(k n, k m+f(\overline{k \bar{n}})), \quad \text { for } k \in K, \\
& {\left[\left(n_{1}, m_{1}\right),\left(n_{2}, m_{2}\right)\right]=\left(\left[n_{1}, n_{1}\right], f\left(\left[\overline{\overline{n_{1}}}, \bar{n}_{2}\right]\right)\right),}
\end{aligned}
$$

where we used the notation

$$
\bar{x}=\eta_{n}(x) \in U G_{n 11}(\Gamma)
$$

for $x \in U G_{n}(\Gamma)$ (see $\$ 1,(1.7)$ ).

Remark. If $\mathfrak{C}={ }_{K} J K$, then $E_{f}=N(\Gamma) \oplus M$ (direct sum) as $K$ - 
module. If $\mathcal{C}=\mathcal{S}^{\times}$, then (4.3) reduces to

$$
\left[\left(n_{1}, m_{1}\right),\left(n_{2}, m_{2}\right)\right]=\left(\left[n_{1}, n_{2}\right], 0\right) .
$$

Define morphisms $\iota: M \rightarrow E_{f}$ and $\varphi: E_{f} \rightarrow G(\Gamma)$ by $\iota(m)=(0, m)$ and $\varphi(n, m)=n$ respectively. Then we have a $U$-split exact sequence in $\mathcal{L}$ :

$$
\left(e_{f}\right): 0 \rightarrow M \stackrel{\iota}{\rightarrow} E_{f} \stackrel{\varphi}{\rightarrow} G(\Gamma) \stackrel{\mathcal{E}}{\rightarrow} \Gamma \rightarrow 0
$$

which will be called a standard two term extension of $\Gamma$ by $M$ (with respect to the underlying object functor $U: \mathcal{L} \rightarrow \mathcal{C}$ ). By (4.3) $M$ is contained in the centre of $E_{f} . G(\Gamma)$ operates on $E_{f}$ by

$$
x(n, m)=([x, n], x m+f([\overline{\bar{x}, \bar{n}}]),
$$

so that we have

$$
\begin{aligned}
& \iota(x m)=x \iota(m), \\
& \varphi(x u)=[x, \varphi(u)]
\end{aligned}
$$

and

$$
\varphi(u) v=[u, v]
$$

for $x \in G(\Gamma), m \in M$ and $u, v \in E_{f}$.

Further we have the following commutative diagram of $K$ modules :

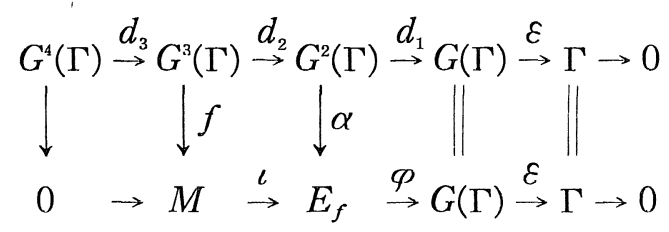

where $\alpha$ is a canonical $K$-linear map $G^{2}(\Gamma) \rightarrow E_{f}$ given as follows. Using the canonical decomposition (see Lemmas 1.4, 1.9)

$$
x=d_{2} s_{1} x+s_{0} d_{1} x, \quad \text { for } \quad x \in G^{2}(\Gamma),
$$

we define $\alpha$ by

$$
\alpha(x)=\left(d_{1} x, f s_{1} x\right) .
$$

Then we can verify that $\alpha$ is $K$-linear.

Proposition 4.12. The K-linear map $\alpha$ satisfies 


$$
\alpha[x, y]=\varepsilon^{0} x \cdot \alpha(y)-\varepsilon^{1} y \cdot \alpha(x) \quad \text { for } \quad x, y \in G^{2}(\Gamma) .
$$

Proof. The right-hand side will be computed by using (4.10), $(4.1 \sim 3)$ as follows :

$$
\varepsilon^{0} x \alpha(y)-\varepsilon^{1} y \cdot \alpha(x)=\left(d_{1}[x, y], m_{1}+m_{2}\right)
$$

where

$$
\begin{aligned}
& m_{1}=\varepsilon^{0} x \cdot f s_{1} y-\varepsilon^{1} y \cdot f s_{1} x, \\
& m_{2}=f\left(\left[\overline{\left.\overline{\varepsilon^{0} x, d_{1} y}\right]-\left[\varepsilon^{1} \bar{y}, \overline{d_{1}} \bar{x}\right.}\right]+\left[\overline{\left.\overline{\varepsilon^{0} x,}, \overline{d_{1} y}\right]-\left[\overline{\varepsilon^{1} y, d_{1} x}\right.}\right]\right) .
\end{aligned}
$$

Since $f$ is a derivation, we have

$$
m_{1}=f\left(\left[\bar{x}, \overline{t_{1} y}\right]+\left[\overline{t_{1} x}, \overline{\delta^{0} \varepsilon^{1} y}\right]+\left[\delta^{0} x, \delta^{0} y\right]\right),
$$

where $t_{1}=1-\delta^{0} \varepsilon^{1}: G^{2}(\Gamma) \rightarrow G^{2}(\Gamma)$ (Cf. (1.5)). Therefore $m_{1}+m_{2}$ is written as $f(\xi)$ for a certain element $\xi \in G^{3}(\Gamma)$, and a direct culculation shows that $d_{2} \xi=d_{2} s_{1}[x, y]$. Since $f$ is a cocycle and $G_{*}^{+}(\Gamma)$ is acyclic as above, we obtain

$$
m_{1}+m_{2}=f(\xi)=f s_{1}[x, y] .
$$

The proposition follows from (4.13) and (4.14).

We have thus assigned canocically the standard two term extension $\left(e_{f}\right)$ to each derivation 2-cocycle $f: G^{3}(\Gamma) \rightarrow M$.

We show that the extension $\left(e_{f}\right)$ belongs to $\varepsilon_{i}$ (see $\S 3$ ). This is clear in case of $\mathcal{C}={ }_{K}$ 秋 or $\mathcal{S}$ by $(4.6 \sim 8)$. In case of $\mathcal{C}=\mathcal{S}^{\times}$, we define a set map $\beta: G(\Gamma) \rightarrow E_{f}$ by

$$
\beta(x)=\alpha \eta_{0}(x)=\alpha(\bar{x}) .
$$

Then, we have, by (1.7)

$$
\phi \beta=\varphi \alpha \eta_{0}=d_{1} \eta_{0}=1-\eta \varepsilon
$$

and by Prop. 4.12

$$
\begin{aligned}
\beta[x, y]=\alpha[\overline{x, y}] & =\alpha[\bar{x}, \bar{y}]=\varepsilon^{0} \bar{x} \cdot \alpha(\bar{y})-\varepsilon^{1} \bar{y} \cdot \alpha(\bar{x}) \\
& =x \beta(y)-\eta \varepsilon y \cdot \beta(x),
\end{aligned}
$$

so that the condition (3.4) is satisfied.

Proposition 4.15. If derivation 2-cocycles $f$ and $f^{\prime}$ are $D$ cohomologous $\left(f \underset{n}{\sim} f^{\prime}\right)$, then we have $\left(e_{f}\right) \sim\left(e_{f^{\prime}}\right)$. 
Proof. Suppose that $f^{\prime}=f+\omega d_{2}$ for a derivation $\omega: G^{2}(\Gamma) \rightarrow M$. Define $\psi: E_{f} \rightarrow E_{f^{\prime}}$ by

$$
\psi(n, m)=(n, m-\omega(\bar{n})) \in E_{f^{\prime}} .
$$

Then $\psi$ is a (bijective) morphism (in $\mathcal{L}$ ) and commutes with the operations of $G(\Gamma)$ on $E_{f}$ and $E_{f^{\prime}}$. We have thus a morphism $\left(e_{f}\right) \rightarrow\left(e_{f^{\prime}}\right)$ in the category $\mathcal{E}_{i}$ of two term extensions of $\Gamma$ by $M$ (see $§ 3)$.

Remark. In a parallel way as above, we can define what to be called the standard $n$ term extension $\left(e_{f}\right)$ of $\Gamma$ by $M$ for derivation $n$-cocycle $f: G^{n+1}(\Gamma) \rightarrow M(n \geq 2)$ as follows.

Define first the Moore subcornplex $\widetilde{G}_{*}(\Gamma)$ of the chain complex $G_{*}(\Gamma)$ of $K$-modules by

$$
\begin{aligned}
& \widetilde{G}_{0}(\Gamma)=G(\Gamma), \\
& \widetilde{G}_{k}(\Gamma)=\bigcap_{i=1}^{k} \operatorname{Ker}\left(\varepsilon^{i}: G^{k+1}(\Gamma) \rightarrow G^{k}(\Gamma)\right) \quad \text { for } k>0 .
\end{aligned}
$$

Then $\widetilde{G}_{k}(\Gamma)$ is an ideal of $G^{k+1}(\Gamma)$ and we have a commutative diagram of $K$-modules :

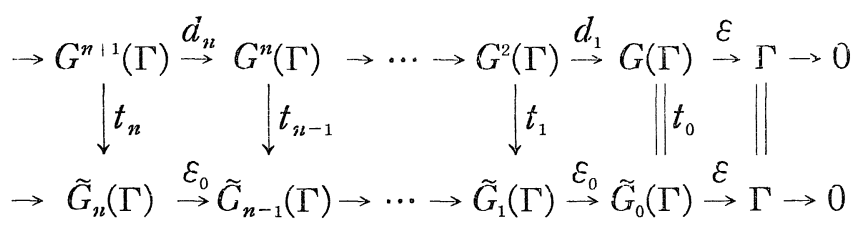

where

$$
t_{n}=\left(1-\delta^{0} \varepsilon^{1}\right)\left(1-\delta^{1} \varepsilon^{2}\right) \cdots\left(1-\delta^{n-1} \varepsilon^{n}\right), \quad n \geq 0
$$

are retractions and define a chain equivalence $G_{*}(\Gamma) \sim \widetilde{G}_{*}(\Gamma)$.

Now let $\widetilde{G}_{n-1}(\Gamma) \times M$ be the direct product of $K$-Lie algebras ( $M$ being an abelian Lie algebra). Let $I$ be the ideal of $\widetilde{G}_{n-1}(\Gamma) \times M$ generated by elements of the form $\left(-t_{n-1} d_{n}(y), f\left(t_{n} y\right)\right)$ for $y \in G^{n+1}(\Gamma)$. Define $E_{f}=\widetilde{G}_{n-1}(\Gamma) \times M / I$ to be the quotient $K$-Lie algebra. Then $E_{f}$ has a canonical set presentation $N_{n-2}(\Gamma) \times M$, where $N_{n-2}(\Gamma)=$ $\operatorname{Ker}\left(\varepsilon^{0}: \widetilde{G}_{n-2}(\Gamma) \rightarrow \widetilde{G}_{n-3}(\Gamma)\right)$ for $n \geq 3$ and $N_{0}(\Gamma)=N(\Gamma)=\operatorname{Ker}(\varepsilon: G(\Gamma) \rightarrow \Gamma)$ as before. Using this set presentation, we can give the $K$-Lie algebra structure of $E_{f}$ explicitly by the same form of formulas as in (4.1), (4.2) and (4.3) (in fact, we have only to replace $N(\Gamma)$ by $N_{n-3}(\Gamma)$ ). 
Then we have a $U$-split exact sequence $\left(e_{f}\right)$ in $\mathcal{L}$ :

$$
0 \rightarrow M \stackrel{\iota}{\rightarrow} E_{f} \stackrel{\rho}{\rightarrow} \widetilde{G}_{n-2}(\Gamma) \stackrel{\varepsilon^{0}}{\rightarrow} \cdots \stackrel{\varepsilon^{0}}{\rightarrow} \widetilde{G}_{0}(\Gamma) \stackrel{\varepsilon}{\rightarrow} \Gamma \rightarrow 0
$$

which we call a standard $n$ term extension of $\Gamma$ by $M$ with respect to the underlying object functor $U$.

Similarly as in (4.10), we have the following commutative diagram

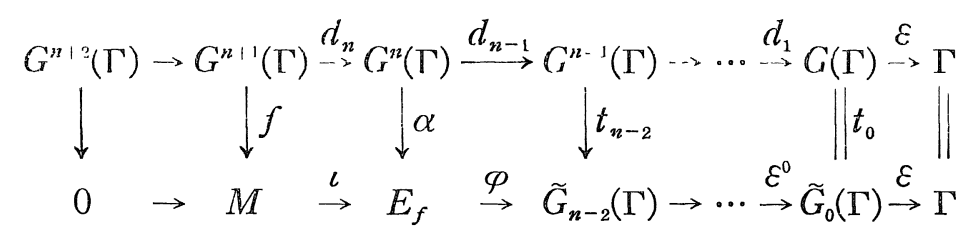

where $\alpha$ is the canonical $K$-map $G^{n}(\Gamma) \rightarrow E_{f}$ explicitly given by

$$
\alpha(x)=\left(t_{n-2} d_{n-1} x, f s_{n-1} x\right) .
$$

\section{\$5. Proof of Theorem 3.5}

In the last section we have defined a map $\Phi: H_{i}^{2}(\Gamma, M) \rightarrow$ $\mathrm{Ex}^{2}(\Gamma, M)(i=1,2,3)$ (see Proposition 4.15). We shall prove the following two propositions of which the first asserts the ontoness of $\Phi$ and the second one asserts $\Phi$ to be 1-1.

Proposition 5.1. Given a two term extension (e) in $\mathcal{E}_{i}(i=1,2,3)$. Then there exists a derivation 2-cocycle $f: G^{3}(\Gamma) \rightarrow M$ such that $\left(e_{f}\right)$ $\rightarrow(e)$ in $\mathcal{E}_{i}$.

Proposition 5.2. If $\left(e_{f}\right)->(e) \leftarrow\left(e_{f^{\prime}}\right)$ in $\mathcal{E}_{i}(i=1,2,3)$, then $f$ and $f^{\prime}$ are D-cohomologous (i.e. $f$ and $f^{\prime}$ determine the same cohomology class $\in H_{i}^{2}(\Gamma, M)$.

Proof Proposition 5.1. Given a two term extension $(e) \in \mathcal{E}_{i}$ :

$$
(e): 0 \rightarrow M \stackrel{\varphi_{2}}{\rightarrow} X_{1} \stackrel{\varphi_{1}}{\rightarrow} X_{0} \stackrel{\mathcal{P}_{0}}{\rightarrow} \Gamma \rightarrow 0
$$

which is $U_{i}$-split exact sequence in $\mathcal{L}$ and $X_{0}$ operates on $X_{1}$ as in (3.1), (3.2) and (3.3) with additional condition (3.4) in case of $\mathcal{C}_{3}=\mathcal{S}^{\times}$.

There exists a morphism $\sigma: U(\Gamma) \rightarrow U\left(X_{0}\right)$ in $\mathcal{C}$ with $\varphi_{0} \sigma=1$, so that $\sigma$ determines a unique morphisin $\tau=\lambda^{-1}(\sigma): G(\Gamma) \rightarrow X_{0}$ in $\mathcal{L}$ by 
(1.1). Then we have

$$
\varphi_{0} \tau=\varepsilon \text {. }
$$

Define the idealization $X_{0} * X_{1}$ of $X_{0}$-module $X_{1}$. That is, $X_{0} * X_{1}$ is the direct sum of $X_{0}$ and $X_{1}$ as $K$-module and the bracket product is given by

$$
\begin{array}{r}
{[(x, u),(y, v)]=([x, y],[u, v]+x \cdot v-y \cdot u)} \\
\text { for } x, y \in X_{0}, u, v \in X_{1} .
\end{array}
$$

We have a morphism $\beta: U\left(X_{0}\right) \rightarrow U\left(X_{1}\right)$ in $\mathcal{C}$ such that

$$
\varphi_{1} \beta=1-\sigma \varphi_{0}
$$

in case of $\mathcal{C}={ }_{K}$ J or $\mathcal{S}$. In case of $\mathcal{C}=\mathcal{S}^{\times}$, we must take such a $\beta$ as in (3.4).

Now define a morphism $\rho^{\prime}: U G(\Gamma) \rightarrow U\left(X_{0} * X_{1}\right)$ in $\mathcal{C}$ by

$$
\rho^{\prime}(x)=(\tau \eta \varepsilon x, \beta \tau x)=\left(\sigma \varphi_{0} \tau x, \beta \tau x\right) \quad \text { for } \quad x \in G(\Gamma) .
$$

This determines a unique morphism $\rho: G^{2}(\Gamma) \rightarrow X_{0} * X_{1}$ in $\mathcal{L}$.

Lemma 5.5. The morphism $\rho: G^{2}(\Gamma) \rightarrow X_{0} * X_{1}$ is expressed by

$$
\rho(x)=\left(\tau \varepsilon^{1} x, g(x)\right),
$$

where $g: G^{2}(\Gamma) \rightarrow X_{1}$ is $K$-linear and satisfies :

and

$$
\varphi_{1} g=\tau d_{1}
$$

for $x, y \in G^{2}(\Gamma)$.

$$
g[x, y]=\tau \varepsilon^{0} x \cdot g(y)-\tau \varepsilon^{1} x \cdot g(x)
$$

Proof. Define two morphisms $\theta^{n}, \theta^{1}: X_{0} * X_{1} \rightarrow X_{0}$ in $\mathcal{L}$ by

$$
\theta^{\circ}(x, u)=x+p_{1}(u)
$$

and

$$
\theta^{1}(x, u)=x
$$

Then using (1.3), we have

$$
\theta^{n} \rho(\bar{x})=\theta^{\circ} \rho^{\prime}(x)=\tau \eta \varepsilon(x)+\varphi_{1} \beta \tau(x)=\tau x=\tau \varepsilon^{n}(\bar{x})
$$

and

$$
\theta^{1} \rho(\bar{x})=\theta^{1} \rho^{\prime}(x)=\tau \eta \varepsilon(x)=\tau \varepsilon^{1}(\bar{x}) .
$$


Using again (1.3), we conclude that

$$
\theta^{0} \rho=\tau \varepsilon^{0}
$$

and

$$
\theta^{1} \rho=\tau \varepsilon^{1}
$$

as morphisms $G^{2}(\Gamma) \rightarrow X_{0}$ in $\mathcal{L}$. The lemma follows from these properties of $\rho$.

Returning to the proof of Prop. 5.1, consider the following commutative diagram :

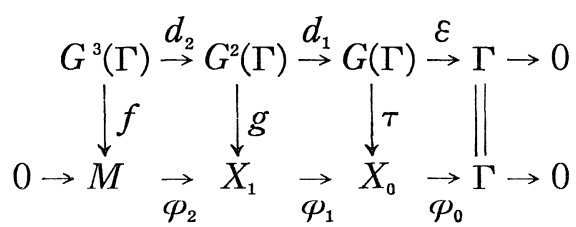

where $f=\varphi_{2}^{-1} g d_{2}$ is a derivation 2-cocycle by Lemma 5.5. We have now a morphism $\left(e_{f}\right) \rightarrow(e)$ in $\mathcal{E}_{i}$ :

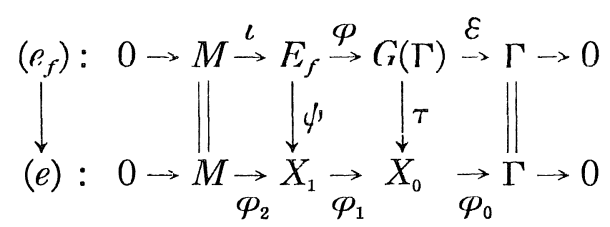

where $\psi: E_{f} \rightarrow X_{1}$ is a morphism in $\mathcal{L}$ defined by

$$
\psi(n, m)=\varphi_{2}(m)+g(\bar{n})
$$

Proof of Proposition 5.2. Suppose that there is given a commutative diagram.

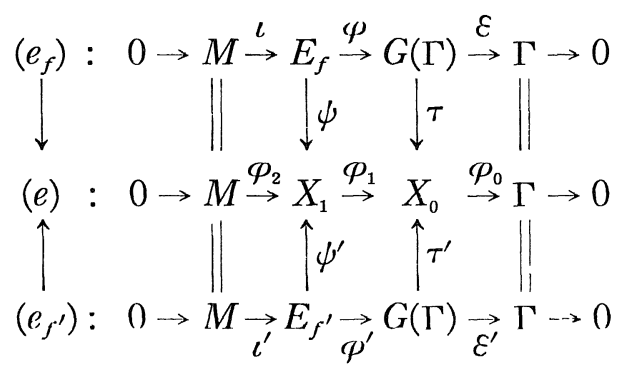

Define a morphism $\rho^{\prime}: U(\Gamma) \rightarrow U\left(X_{0} * X_{1}\right)$ by

$$
\rho^{\prime}(x)=\left(\tau(\bar{x}), \beta \tau^{\prime}(\bar{x})\right) \text {. }
$$


Then $\rho^{\prime}$ determines a unique morphism $\rho: G(\Gamma) \rightarrow X_{0} * X_{1}$ in $\mathcal{L}$.

Lemma 5.6. The morphism $\rho$ is expressible in the form

$$
\rho(x)=(\tau(x), \omega(x)),
$$

where $\omega: G(\Gamma) \rightarrow X_{1}$ is K-linear and satisfies:

$$
\varphi_{1} \omega=\tau^{\prime}-\tau
$$

and

$$
\omega[x, y]=\tau^{\prime}(x) \cdot \omega(y)-\tau(y) \cdot \omega(x), \quad \text { for } \quad x, y \in G(\Gamma) .
$$

The proof is similar as in that of Lemma 5.5 and hence ommited. Now consider a $K$-linear map

$$
\omega_{1}=\psi^{\prime} \alpha^{\prime}-\psi \alpha-\omega d_{1}: \quad G^{2}(\Gamma) \rightarrow X_{1},
$$

where $\alpha^{\prime}: G^{2}(\Gamma) \rightarrow E_{f^{\prime}}$ and $\alpha: G^{2}(\Gamma) \rightarrow E_{f}$ are defined in (4.11). Then

$$
\varphi_{1} \omega_{1}=\tau^{\prime} \varphi^{\prime} \alpha^{\prime}-\tau \varphi \alpha-\left(\tau^{\prime}-\tau\right) d_{1}=0 .
$$

Therefore $\omega_{1}$ is regarded as a map : $G^{2}(\Gamma) \rightarrow M$. A straightforward calculation shows that

$$
\begin{aligned}
\omega_{1}[x, y] & =\tau^{\prime} \varepsilon^{0} x \cdot \omega_{1}(y)-\tau \varepsilon^{1} y \cdot \omega_{1}(x) \\
& =\varepsilon \varepsilon^{0} x \cdot \omega_{1}(y)-\varepsilon \varepsilon^{1} y \cdot \omega_{1}(x)
\end{aligned}
$$

This means that $\omega_{1}: G^{2}(\Gamma) \rightarrow M$ is a derivation. And clearly we have

$$
\varphi_{2} \omega_{1} d_{2}=\psi^{\prime} \alpha^{\prime} d_{2}-\psi^{\prime} \alpha d_{2}=\psi^{\prime} \iota^{\prime} f^{\prime}-\psi \iota f=\varphi_{2}\left(f^{\prime}-f\right),
$$

that is, $\omega_{1} d_{2}=f^{\prime}-f$.

q.e.d.

\section{References}

[1] Barr, M. and J. Beck, Acyclic models and triples, Proceedings of the La Jolla Conference on Categorical Algebra, Springer, 1966, pp. 336-343.

[2] - Shukla cohomology and triples, J. of Algebra 5 (1967), 222-231.

[3] - Harrison homology, Hochschild homology and triples, J. of Algebra 8 (1968), 314-323.

[4] - Cohomology and obstructions: commutative algebras, Seminar on Triples and Categorical Homology Theory, Lecture Notes in Mathematics No. 80, Springer, 1969, pp. 357-375.

[5] Beck, J., Triples, Algebras and Cohomology, Doctoral dissertation, Columbia University, 1967.

[6] Cartan, H. and S. Eilenberg, Homological Algebra, Princeton University Press, 1956. 
[7] Dixmier, J., Homologic des anneaux de Lie, Anı. Sci. Ecole Norn. Sup. 74 (1957), 25-83.

[8] Dold, A., S. MacLane and U. Obersl, Projective Classes and Acyclic Models, Reports of the Midwest Categorical Seminar, Lecture Notes in Mathematics No. 47, Springer, 1967, pp. 78-91.

[9] Dubuc, E., Adjoint triangles, Reports of the Midwest Categorical Seminar II, Lecture Notes in Mathematics No. 61, Springer, 1968, pp. 69-91.

[10] Eilenberg, S. and S. MacLane, Cohomology theory in abstract groups II. Group extensions with a non-abelian kernel, Ann. of Math. 48 (1947), 326-341; S. MacLane, Cohomology theory in abstract groups III, Operator homomorphisms of kernels, Ibid. 50 (1949), 736-761

[11] Eilenberg, S. and J. C. Moore, Foundation of Relative Homological Algebra, Memoirs Amer. Math. Soc., No. 55, 1965.

[12] — Adjoint functors and triples, Illinois J. Math. 9 (1965), 381-398.

[13] Gerstcnhaber, M., On the deformation of rings and algebras II, Ann. of Matlı. 84 (1966), 1-19.

[14] - The third cohomology group of a ring and the commutative cohomology theory, Bull. Amer. Math. Soc. 73 (1967) 950-954.

[15] Godcment, R., Théorie des Faisceaux, Hermann, Paris, 1958.

[16] Harrison, D. K., Commutative algebras and cohomology, Trans. Amer. Math Soc. 104 (1962), 191-204.

[17] Hochschild, G., On the cohomology groups of an associative algebra, Ann. of Math. 46 (1945), 58-67.

[18] - Cohomology and representation of associative algebras, Duke Math. J. 11 (1947), 921-948.

[19] Huber, P. J., Homotopy theory in general categorics, Math. Ann. 144 (1961), 361-385.

[20] Iwai, A., On the standard complexes of cotriples, Proc. Japan Acad. 10 (1968), 327-329.

[21] - Simplicial cohomology and $n$ term extensions of algebras, to appear.

[22] Kleisli, H., Every standard construction is induced by a pair of adjoint functors, Proc. Amer. Math. Soc. 16 (1965), 544-546.

[23] Lichtenbaum, S. and S. Schlessinger, The cotangent complex of a morphism, Trans. Amer. Math. Soc. 128 (1967), 41-70.

[24] MacLane, S., Extension and obstructions for rings, Illinois J. Math. 2 (1958), $316-345$.

[25] - Homology, Springer and Academic Press, 1963.

[26] Shimada, N., H. Uehara and F. Brenneman, Triple cohomology in relative homological algebra, Notices of Amer. Math. Soc. 14 (1967), 659.

[27] Shukla, U., Cohomologie des algèbres associatives, Ann. Sci. Ecole Norm. Sup. 78 (1961), 163-209.

[28] - A cohomology for Lie algebras, Math. Soc. Japan, 18 (1966), 275289.

[29] Yoneda, N., On the homology theory of modules, J. Fac. Sci. Tokyo, Sec. I, 7 (1954), 193-227. 
